\title{
Fault Detection and Isolation Scheme Based on Parity Space Method for Discrete Time-Delay System
}

\author{
Hongyu Wang, Zuohua Tian, Songjiao Shi and Zhenxin Weng \\ Shanghai Jiaotong University
}

China

\section{Introduction}

In recent years, fault detection and isolation (FDI) problem in dynamitic system has been paid more and more attention. A great number of methods for FDI have been proposed (Chow \& Willsky, 1984; Frank \& Ding, 1997; Chen \& Patton, 1999; Patton et al., 2000; Venkatasubramanian et al., 2003). All of the FDI schemes are concerned with a core stage: the generation of the residual signals. The difference between the measurement of the system and its estimation is called residual, whose values are zero or near to zero when no fault occurs while differ distinctly from zero otherwise. Appropriate decisions such as the occurrence, magnification, type, location, etc. of the faults are called fault isolation, which are achieved by residual evaluation.

In the field of analytical model-based FDI techniques, the analytical redundancy relations of the system are used to create residual signal. The approaches can be roughly classified into observer-based approaches and parameter estimation approaches. Parity space approaches have been proved to be structurally equivalent to the observer-based though the design procedures differ (Gertler, 1991). However, the parity space methodology using the temporal redundancy has its advantages, especially in the discrete system. This method was firstly generalized by the (Chow \& Willsky, 1984).

Time delays are inherent in many real physical processes (i.e. mechanical and chemical processes, long transmission lines in pneumatic systems, power and water distribution networks, air pollution systems etc.) Over the past two decades, analysis and synthesis of dynamic time-delay systems have attracted a great deal of interests (Dugard \& Verriest, 1997; Yang \& Saif, 1998). However, there are relative fewer research results on FDI of timedelay systems (KRATZ et al., 1998; Zhong et al., 2004).

This paper proposes a method to deal with the FDI problem for the linear discrete-time systems with delays. The results in (KRATZ et al., 1998) are extended. Both fault detection and fault isolation method are proposed. The occurrence of the fault can be detected timely and the position of the fault can be located exactly. A numerical example is given to illustrate the design method at the end. 


\section{Mathematical Preliminaries}

A time delay operator $\nabla$ is defined according to (KRATZ et al., 1998). $\nabla f(k)=f(k-1)$ for any discrete-time function $f$. It is easy to understand that $\nabla \nabla f(k)=\nabla^{2} f(k)=f(k-2)$, $\nabla^{w} f(k)=f(k-w)$.

Consider a linear discrete time-delay system described by

$$
\left\{\begin{array}{l}
\boldsymbol{x}(k+1)=\sum_{i=0}^{v} \boldsymbol{A}_{i} \boldsymbol{x}(k-i)+\boldsymbol{B}\left[\boldsymbol{u}(k)+\boldsymbol{f}_{a}(k)\right]+\boldsymbol{E}_{d} \boldsymbol{d}(k) \\
\boldsymbol{y}(k)=\boldsymbol{C} \boldsymbol{x}(k)+\boldsymbol{F}_{d} \boldsymbol{d}(k)
\end{array}\right.
$$

where $\boldsymbol{x}(k) \in R^{n}$ is the state vector, $\boldsymbol{u}(k) \in R^{p}$ is the control vector, $\boldsymbol{y}(k) \in R^{m}$ is the output vector, $\boldsymbol{f}_{a}(k)=\left[f_{a 1}(k), \cdots, f_{a p}(k)\right]^{\mathrm{T}}$ stands for the actuator faults. $f_{a i}(k), i=1, \cdots, p$ is corresponding to the $i$ th actuator fault. $\boldsymbol{d}(k) \in R^{l}$ is disturbance vector, $\|\boldsymbol{d}(k)\|<\bar{d} . \boldsymbol{A}_{i}(i=0, \cdots, v), \boldsymbol{B}, \boldsymbol{C}, \boldsymbol{E}_{d}$ and $\boldsymbol{F}_{d}$ are constant matrices with appropriate dimensions. Integers $v \geq 0$ denotes the number of time delays in the state.

Using the operator $\nabla$, the system (1) can be rewritten as

$$
\left\{\begin{array}{l}
\boldsymbol{x}(k+1)=\boldsymbol{A}(\nabla) \boldsymbol{x}(k)+\boldsymbol{B}\left[\boldsymbol{u}(k)+\boldsymbol{f}_{a}(k)\right]+\boldsymbol{E}_{d} \boldsymbol{d}(k) \\
\boldsymbol{y}(k)=\boldsymbol{C} \boldsymbol{x}(k)+\boldsymbol{F}_{d} \boldsymbol{d}(k)
\end{array}\right.
$$

where,

$$
\boldsymbol{A}(\nabla)=\boldsymbol{A}_{0}+\boldsymbol{A}_{1} \nabla+\cdots+\boldsymbol{A}_{v} \nabla^{v}
$$

\section{Parity Space Residual Generation for Fault Detection and Isolation}

The task of FDI is to design a residual signal which is zero or near to zero in a fault free case and non-zero when a fault occurs in the monitored system. Time delay implies that the state of the system for the next time step is not only determined by the current state but also concerned with the state of the former intervals. The recursion of equation (2) from time instant $k-L$ to time instant $k$ yields

$$
\boldsymbol{y}_{L}=\boldsymbol{H}_{o, L} \boldsymbol{x}(k-L)+\boldsymbol{H}_{u, L} \boldsymbol{u}_{L}+\boldsymbol{H}_{d, L} \boldsymbol{d}_{L}+\boldsymbol{H}_{f a, L} \boldsymbol{f}_{a L}
$$

where 


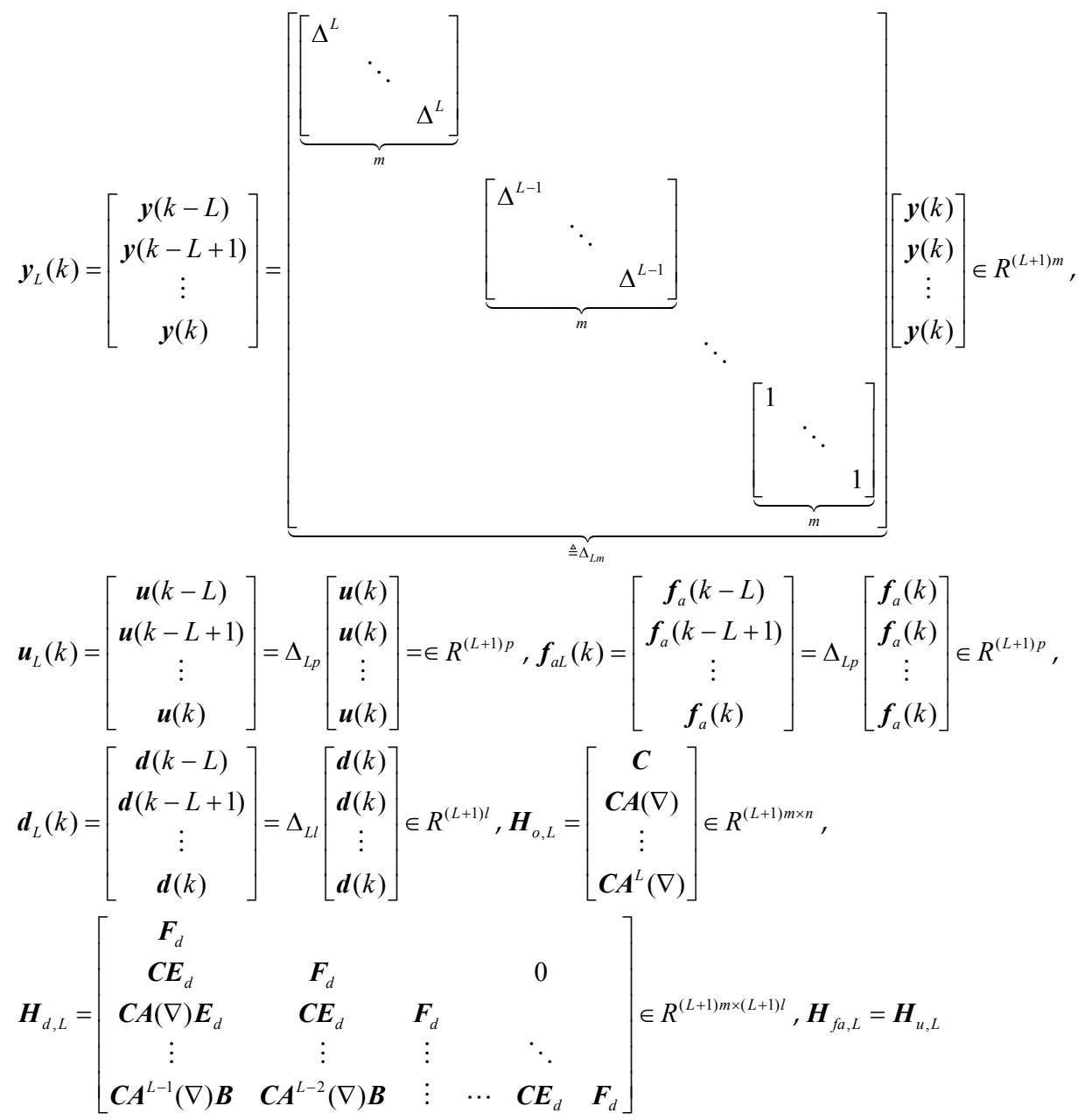

Define the following parity space:

$$
\boldsymbol{P}_{L}=\left\{\boldsymbol{v}_{L} \mid \boldsymbol{v}_{L} \boldsymbol{H}_{o, L}=0\right\}
$$

where $\boldsymbol{v}_{L} \in R^{1 \times(L+1) m}$ is row vector. Vectors belong to parity space are called parity vectors. Residual signals can be created by the folowing equation:

$$
r_{L}(k)=\boldsymbol{v}_{L}\left[\boldsymbol{y}_{L}(k)-\boldsymbol{H}_{u, L} \boldsymbol{u}_{L}(k)\right] \in \boldsymbol{R}
$$

Substituting equation (6) to equation (4) yields:

$$
r_{L}(k)=\boldsymbol{v}_{L} \boldsymbol{H}_{d, L} \boldsymbol{d}_{L}(k)+\boldsymbol{v}_{L} \boldsymbol{H}_{f a, L} \boldsymbol{f}_{a L}(k)
$$


It should be noted that the parity vectors $\boldsymbol{v}_{L}$ satisfying equation (5) are not unique, and the corresponding residual signals $r_{L}(k)$ are not unique. The freedom of the $\boldsymbol{v}_{L}$ can be used to creat specific residual signals, so as to fulfill specific design purpose.

The parity vectors $\boldsymbol{v}_{L}$ can be described as $\boldsymbol{v}_{L}=\left[v_{1}, v_{2}, \cdots, v_{(L+1) m}\right]$. Substituting it to equation (7), the terms corresponding to disturbance $\boldsymbol{v}_{L} \boldsymbol{H}_{d, L} \boldsymbol{d}_{L}(k)$ and faults $\boldsymbol{v}_{L} \boldsymbol{H}_{f a, L} \boldsymbol{f}_{a L}(k)$ can be respectively expanded as follows:

$$
\begin{aligned}
& \boldsymbol{v}_{L} \boldsymbol{H}_{d, L} \boldsymbol{d}_{L}(k)=\psi_{1} d_{1}(k)+\psi_{2} d_{2}(k)+\cdots+\psi_{l} d_{l}(k) \\
& \boldsymbol{v}_{L} \boldsymbol{H}_{f a, L} \boldsymbol{f}_{a L}(k)=\omega_{1} f_{a 1}(k)+\omega_{2} f_{a 2}(k)+\cdots+\omega_{p} f_{a p}(k)
\end{aligned}
$$

Where $\psi_{1}, \psi_{2}, \cdots, \psi_{p}, \omega_{1}, \omega_{2}, \cdots, \omega_{p}$ are polynomials corresponding to $v_{1}, v_{2}, \cdots, v_{(L+1) m}$ and $\nabla$.

The successful detection of a fault is followed by the fault isolation procedure which will distinguish (isolate) a particular fault from others. While a single residual signal is sufficient to detect faults, a set of residuals (or a vector of residual) is usually required for fault isolation. According to (Chen \& Patton, 1999), a commonly used scheme in designing the residual set is to make each residual sensitive to all but one fault, i.e.

$$
\left\{\begin{array}{l}
r_{1}=R\left(f_{a 2}, \cdots, f_{a 3}\right) \\
\quad \vdots \\
r_{i}=R\left(f_{a 1}, \cdots, f_{a i-1}, f_{a i+1}, \cdots, f_{a p}\right) \\
\quad \vdots \\
r_{p}=R\left(f_{a 1}, \cdots, f_{a p-1}\right)
\end{array}\right.
$$

where $R(\cdot)$ denotes some functional relation, which works as the residual generator. This is defined as a generalized structured residual set. The isolation can be performed by the following logic:

$$
\left.\begin{array}{l}
\left\|r_{i}\right\| \leq t h^{i} \\
\left\|r_{j}\right\|>t h^{j} \text { for } j=1, \cdots, i-1, i+1, \cdots p
\end{array}\right\} \Rightarrow f_{a i} \neq 0
$$

where $t h^{i}$ means the fault isolation threshold to the corresponding fault.

To achieve the so called generalized structured residual set, let $\omega_{1}, \omega_{2}, \cdots, \omega_{p}$ satisfy the folowing equatinos:

$$
\left\{\begin{array}{c}
\omega_{1} \neq 0 \\
\vdots \\
\omega_{i-1} \neq 0 \quad, i=1,2, \cdots, p \\
\omega_{i}=0 \\
\omega_{i+1} \neq 0
\end{array}\right.
$$

Solving the equations (12) respectively can achieve a set of parity vecters $\boldsymbol{v}_{L f a i} i=1,2, \cdots, p$, witch lead to a set of residual signals $r_{L f a i}(k), i=1,2, \cdots, p$ by equation (6). When the $i$ th actuator fault occurs $\left(f_{a i}(k) \neq 0\right)$, the corresponding residual signal $r_{L f a i}(k)$ is not affected, while the other residual signals $r_{L f a 1}(k), r_{L f f a 1}(k) ; \cdot \cdot, r_{L f a i-1}(k), r_{L f a i+1}(k), r_{L f a p}(k)$ are affected. The isolation can be fulfilled by equaton (11). 


\section{Numerical Example}

To illustrate the design process of the proposed mothed and verify its effectiveness, the following numerical example is demonstrated. Consider a time delay system of the form (l),

$$
\begin{aligned}
& \left(\left[\begin{array}{l}
x_{1}(k+1) \\
x_{2}(k+1) \\
x_{3}(k+1)
\end{array}\right]=\left[\begin{array}{ccc}
0.15 & 0.324 & 0 \\
0.102 & 0.258 & 0 \\
0 & 0 & 0.412
\end{array}\right]\left[\begin{array}{l}
x_{1}(k) \\
x_{2}(k) \\
x_{3}(k)
\end{array}\right]+\left[\begin{array}{ccc}
0 & 0.265 & 0 \\
-0.406 & 0 & 0.308 \\
0 & -0.252 & 0.124
\end{array}\right]\left[\begin{array}{l}
x_{1}(k-1) \\
x_{2}(k-1) \\
x_{3}(k-1)
\end{array}\right]\right. \\
& +\left[\begin{array}{ccc}
1 & 0 & 0 \\
0 & 1 & 0 \\
0 & 0 & 1
\end{array}\right]\left(\left[\begin{array}{l}
u_{1}(k) \\
u_{2}(k) \\
u_{3}(k)
\end{array}\right]+\left[\begin{array}{l}
f_{a 1}(k) \\
f_{a 2}(k) \\
f_{a 3}(k)
\end{array}\right]\right)+\left[\begin{array}{l}
1 \\
1 \\
1
\end{array}\right] d(k) \\
& {\left[\begin{array}{l}
y_{1}(k) \\
y_{2}(k) \\
y_{3}(k)
\end{array}\right]=\left[\begin{array}{lll}
1 & 0 & 0 \\
0 & 1 & 0 \\
0 & 0 & 1
\end{array}\right]\left[\begin{array}{l}
x_{1}(k) \\
x_{2}(k) \\
x_{3}(k)
\end{array}\right]+\left[\begin{array}{l}
1 \\
1 \\
1
\end{array}\right] d(k)}
\end{aligned}
$$

Where $\boldsymbol{d}(k)=0.01 \times \operatorname{rand} N(0,1), \operatorname{rand} N(0,1)$ stands for zero mean, uint Gauss noise. Using the operator $\nabla$, the system (1) can be rewritten into the form (2), the system matrix is:

$$
\boldsymbol{A}(\nabla)=\left[\begin{array}{ccc}
0.15 & 0.324+0.265 \Delta & 0 \\
0.102-0.406 \Delta & 0.258 & 0.308 \Delta \\
0 & -0.252 \Delta & 0.412+0.124 \Delta
\end{array}\right]
$$

In order to fulfill the FDI, a parity space with $L=2$ is established, the matrix $\mathbf{H}_{o, L}, \boldsymbol{H}_{u, L}$, etc. can be computed using the software MAPLE. Solving the equation (5) can get the parity vecter:

$$
v_{L}=\left[\begin{array}{c}
t_{1} \\
\left(\begin{array}{c}
1.767 \nabla t_{1}+2.160 t_{1}-0.03768 t_{2}-0.7173 \nabla^{2} t_{2}-0.6968 \nabla t_{2}+0.2520 \nabla t_{3} \\
-0.01221 t_{4}-0.2357 \nabla t_{4}-0.1901 \nabla^{3} t_{4}-0.4170 \nabla^{2} t_{4}-0.1074 \nabla^{2} t_{5}-0.009721 t_{5} \\
-0.1798 \nabla t_{5}+0.1133 \nabla t_{6}+0.2068 \nabla^{2} t_{6}+0.1807512 \nabla^{3} t_{6}
\end{array}\right) \\
\left(\begin{array}{l}
-0.308 \nabla t_{2}-0.412 t_{3}-0.124 \nabla t_{3}-0.09979 \nabla t_{4}-0.08162 \nabla^{2} t_{4}-0.2064 \nabla t_{5}- \\
0.03819 \nabla^{2} t_{5}+0.06224 \nabla^{2} t_{6}-0.1023 \nabla t_{6}-0.1697 t_{6}
\end{array}\right) \\
-6.667 t_{1}-0.6800 t_{2}+2.7067 \nabla t_{2}-0.3703 t_{4}+0.6968 \nabla t_{4}+0.7173 \nabla^{2} t_{4}+1.104 \nabla t_{5} \\
-0.2774 t_{5}-0.6821 \nabla^{2} t_{6}+0.1714 \nabla t_{6} \\
t_{2} \\
t_{3} \\
t_{4} \\
t_{5} \\
t_{6}
\end{array}\right]
$$

Where $t_{1}, t_{2}, t_{3}, t_{5}, t_{5}, t_{6} \in R$ and $t_{1}, t_{2}, t_{3}, t_{5}, t_{5}, t_{6}$ are not all zeros. $v_{L}$ is a row 9 dimensions vector with 6 fredom. 
In order to achieve fault detection and isolation, a generalized residual set consists in three residual signals should be created as show in Fig.1.

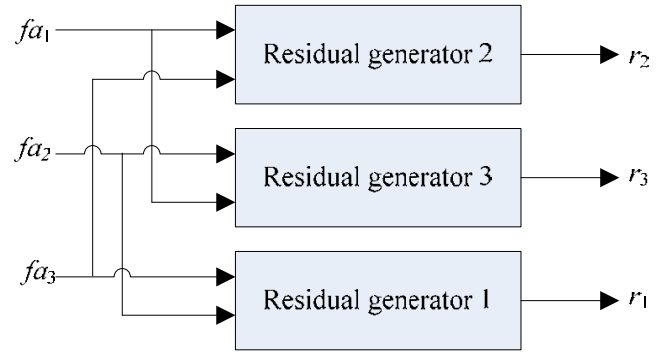

Fig. 1 Generalized residual set

In Fig.1, $f_{a i}$ stands for the $i$ th actuator fault. Residual singal $r_{1}$ is sensitive to $f_{a 2}$ and $f_{a 3}$ while insensitive to $f_{a 1}$. The situation of $r_{2}$ and $r_{3}$ are the same with $r_{1}$.

Substituting $v_{L}$ to equation (7), and expanding the terms corresponding to the actuator fault $\boldsymbol{v}_{L} \boldsymbol{H}_{f a, L} \boldsymbol{f}_{a L}(k)$ into equation (9) yields, $\boldsymbol{v}_{L} \boldsymbol{H}_{f a, L} \boldsymbol{f}_{a L}(k)=\omega_{1} f_{a 1}(k)+\omega_{2} f_{a 2}(k)+\omega_{3} f_{a 3}(k)$. Where $\omega_{1}, \omega_{2}, \omega_{3}$ are polynomials corresponding to $t_{1}, t_{2}, t_{3}, t_{5}, t_{5}, t_{6}$ and $\nabla$. Using equation (12), let

$$
\left\{\begin{array}{l}
\omega_{1}=0 \\
\omega_{2} \neq 0 \\
\omega_{3} \neq 0
\end{array}\right.
$$

$t_{1}, t_{2}, t_{3}, t_{5}, t_{5}, t_{6}$ can be achieved by solving the above equation, and substituting them into (13) can get the parity vector corresponding to the first actuator fault $f_{a 1}$ :

$$
v_{L f a 1}=\left[\begin{array}{c}
0 \\
0.2520 \Delta+0.07762 \Delta^{2} \\
-0.4120-0.2509 \Delta-0.03819 \Delta^{2} \\
-0.1020+0.4060 \Delta \\
-0.2580 \\
1 \\
0 \\
1 \\
0
\end{array}\right]^{T}
$$

Substituting $v_{L f a 1}$ to equation (6) can get generalized residual signal $r_{L f f 1}(k)$ corresponding to $f_{a 1}$, denoted by $r_{1}$.

$r_{1}=\left(-0.1020 \Delta+0.40600 \Delta^{2}\right) y_{1}(k)+\left(1-0.2580 \Delta+0.2520 \Delta^{3}+0.07762 \Delta^{4}\right) y_{2}(k)+$

$\left(\Delta-0.4120 \Delta^{2}-0.2509 \Delta^{3}-0.03819 \Delta^{4}\right) y_{3}(k)+(-\Delta) u_{2}(k)+\left(-\Delta^{2}-0.3080 \Delta^{3}\right) u_{3}(k)$

By the same process, let 


$$
\left\{\begin{array}{l}
\omega_{1} \neq 0 \\
\omega_{2}=0 \\
\omega_{3} \neq 0
\end{array},\left\{\begin{array}{l}
\omega_{1} \neq 0 \\
\omega_{2} \neq 0 \\
\omega_{3}=0
\end{array}\right.\right.
$$

The residual signals correspond to $f_{a 2}$ and $f_{a 3}$ can be designed, denoted by $r_{2}$ and $r_{3}$.

Now, the disign of the generalized residual set for actuator fault detection and isolation is accomplished. The result of the simulation is show in Fig. 2 to Fig. 5.

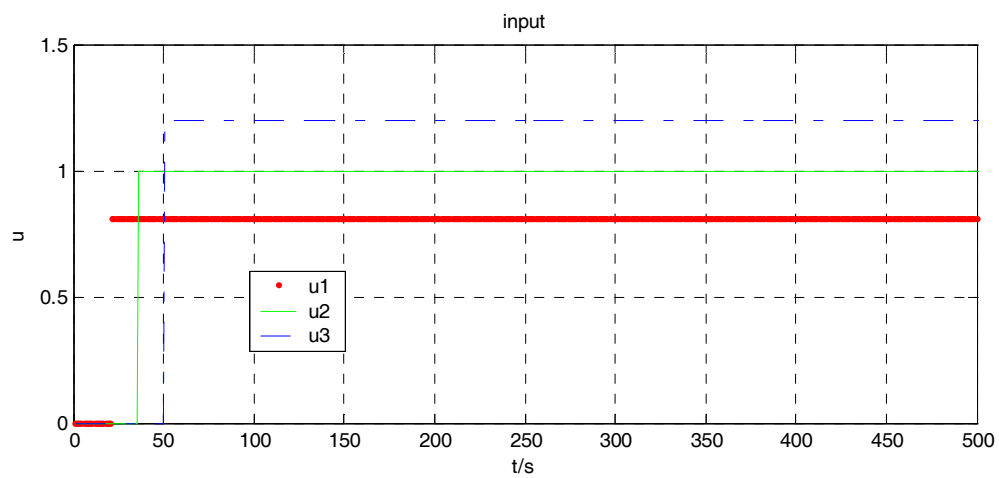

Fig. 2 System input signals

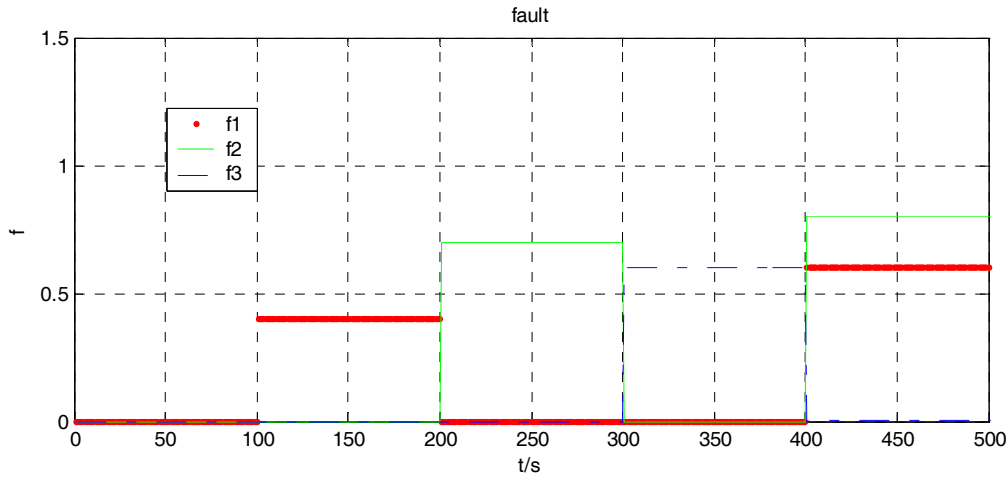

Fig. 3 Fault signals 


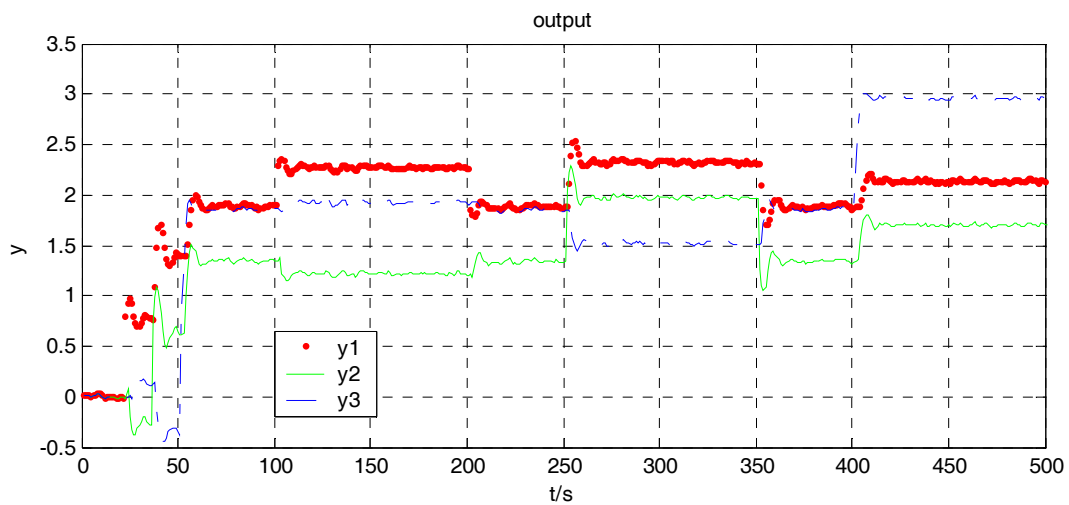

Fig. 4 System output signals

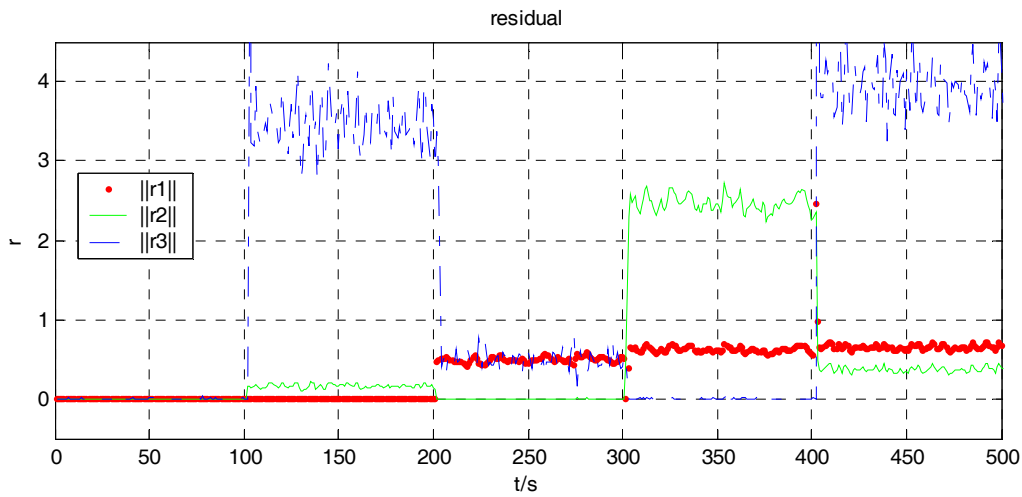

Fig. 5 Residual signals

There are 5 stages in the simulation process:

1. from time 0 second to time 100 second, the system works properly. $\left\|r_{1}\right\|,\left\|r_{2}\right\|$ and $\left\|r_{3}\right\|$ are near to zreo.

2. from time 100 second to time 200 second, the actuator 1 suffers from fault, while actuator 2 and actuator 3 work properly. $\left\|r_{2}\right\|$ and $\left\|r_{3}\right\|$ differ from zero while $\left\|r_{1}\right\|$ keeps zero nearby.

3. from time 200 second to time 300 second, the actuator 2 suffers from fault, while actuator 1 and actuator 3 work properly. $\left\|r_{1}\right\|$ and $\left\|r_{3}\right\|$ differ from zero while $\left\|r_{2}\right\|$ keeps zero nearby.

4. from time 300 second to time 400 second, the actuator 3 suffers from fault, while actuator 1 and actuator 2 work properly. $\left\|r_{1}\right\|$ and $\left\|r_{2}\right\|$ differ from zero while $\left\|r_{3}\right\|$ keeps zero nearby.

5. from time 400 second to time 500 second, the actuator 1 and actuator 2 suffer from fault at the same time, while actuator 3 works properly. $\left\|r_{1}\right\|,\left\|r_{2}\right\|$ and $\left\|r_{3}\right\|$ differ from zero simultaneously. 
It can be concluded that when there is one actuator goes into fault, the above generalize residual set based on pariy space can detect the fault and isolate which actuator corrupted by the fault. However, when there are more than one actuators break into faults, the method can only detect the fault, while it have no idea that which actuators corrupted by the fault.

\section{Conclusion}

A fault detection and isolation scheme for discrete time-delay system has been proposed in this chapter. The scheme can not only detect the faults but also isolate (locate) the faults. To fulfill the FDI, a generalized residual set in form of parity space is designed by the recursion of the system equations. Each residual is sensitive to all but one actuator faults. The actuator with fault can be isolated from the normal ones exactly. A time delay operator is used to deal with the problem brought by the time-delay system. The effectiveness of the proposed method has been verified by a numerical example.

However, further studies are required which include the follow aspects:

1. To determine an optimal recursion step $L$. Such that the residuals can obtain a certain freedom to complete fault isolation, while the computation is minimized.

2. To extend the fault isolation result. The sensor faults and the actuator faults should be discerned.

3. To enhance the reliability and robust performance of the FDI system.

\section{References}

Chen, J.; Patton, R. J. (1999). Robust Model-based Fault Diagnosis for Dynamic Systems, Kluwer Academic Publishers

Chow, E. Y.; Willsky, A. S. (1984). Analytic redundancy and the design of robust fault detection systems. IEEE Transaction on Automatic Control, Vol. 29, Jul. 1984, 603-614

Dugard, L.; Verriest, E. I. (1997). Stability and Control of Time-delay Systems. Lecture Notes in Control and Information Sciences, Springer

Frank, P. M.; Ding, X. (1997). Survey of robust residual generation and evaluation methods in observer-based fault detection systems. Journal of Process Control, Vol. 7, Jun. 1997 pp. $403-424$

Gertler, J. Li, B. (1991). Analytical redundancy methods in fault detection and isolation, Proceedings of the IFACIIMACS Symposium SAFEPROCESS 9, pp. 9-21, Baden-Baden 1991

KRATZ, F.; NUNINGER, W. \& PLOIX, S. (1998). Fault detection for time-delay systems: a parity space approach, Proceedings of the American Control Conference, pp. 2009-2011, Philadelphia, Pennsylvania, 1998

Patton, R. J.; Frank, P. M. and Clark, R. N. (2000). Issues of Fault Diagnosis for Dynamic Systems, Springer

Venkatasubramanian, V.; Rengaswamy, R.; Yin, K. and Kavuri, Surya N. (2003). S A review of process fault detection and diagnosis: Part I: quantitative model-based methods. Computers and Chemical Engineering, Vol. 27, 2003 pp. 293-311

Yang, H.; Saif, M. (1998). Observer design and fault diagnosis for state retarded dynamical systems. Automatica, Vol. 34, Feb. 1998 pp. 217-227 
Zhong, M.; Ye, H. \& Wang, G. (2004). Multi-freedom design of fault detection filter for linear time-delay systems, Proceedings of 2004 8th Inernational Conference on Control, Automation, Robotics and Vision, pp. 1630-1634, Kunming, China, 2004 


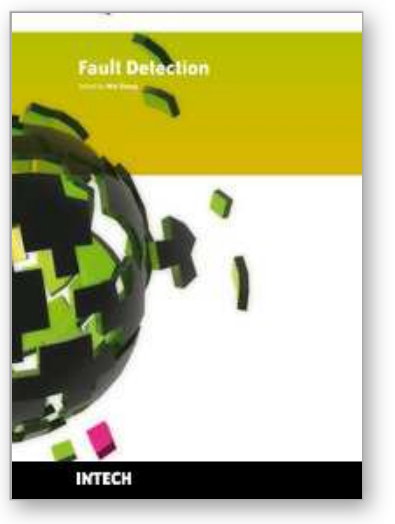

\author{
Fault Detection \\ Edited by Wei Zhang
}

ISBN 978-953-307-037-7

Hard cover, 504 pages

Publisher InTech

Published online 01, March, 2010

Published in print edition March, 2010

In this book, a number of innovative fault diagnosis algorithms in recently years are introduced. These methods can detect failures of various types of system effectively, and with a relatively high significance.

\title{
How to reference
}

In order to correctly reference this scholarly work, feel free to copy and paste the following:

Hongyu Wang, Zuohua Tian, Songjiao Shi and Zhenxin Weng (2010). Fault Detection and Isolation Scheme Based on Parity Space Method for Discrete Time-Delay System, Fault Detection, Wei Zhang (Ed.), ISBN: 978953-307-037-7, InTech, Available from: http:/www.intechopen.com/books/fault-detection/fault-detection-andisolation-scheme-based-on-parity-space-method-for-discrete-time-delay-system

\section{INTECH}

open science | open minds

\section{InTech Europe}

University Campus STeP Ri

Slavka Krautzeka 83/A

51000 Rijeka, Croatia

Phone: +385 (51) 770447

Fax: +385 (51) 686166

www.intechopen.com

\section{InTech China}

Unit 405, Office Block, Hotel Equatorial Shanghai

No.65, Yan An Road (West), Shanghai, 200040, China 中国上海市延安西路65号上海国际贵都大饭店办公楼 405 单元

Phone: +86-21-62489820

Fax: +86-21-62489821 
(C) 2010 The Author(s). Licensee IntechOpen. This chapter is distributed under the terms of the Creative Commons Attribution-NonCommercialShareAlike-3.0 License, which permits use, distribution and reproduction for non-commercial purposes, provided the original is properly cited and derivative works building on this content are distributed under the same license. 